\title{
Near-wake flow structure downwind of a wind turbine in a turbulent boundary layer
}

\author{
Wei Zhang · Corey D. Markfort • Fernando Porté-Agel
}

Received: 10 May 2011/Revised: 29 November 2011/Accepted: 7 December 2011/Published online: 21 December 2011

(C) Springer-Verlag 2011

\begin{abstract}
Wind turbines operate in the surface layer of the atmospheric boundary layer, where they are subjected to strong wind shear and relatively high turbulence levels. These incoming boundary layer flow characteristics are expected to affect the structure of wind turbine wakes. The near-wake region is characterized by a complex coupled vortex system (including helicoidal tip vortices), unsteadiness and strong turbulence heterogeneity. Limited information about the spatial distribution of turbulence in the near wake, the vortex behavior and their influence on the downwind development of the far wake hinders our capability to predict wind turbine power production and fatigue loads in wind farms. This calls for a better understanding of the spatial distribution of the 3D flow and coherent turbulence structures in the near wake. Systematic wind-tunnel experiments were designed and carried out to characterize the structure of the near-wake flow downwind of a model wind turbine placed in a neutral boundary layer flow. A horizontal-axis, three-blade wind turbine model, with a rotor diameter of $13 \mathrm{~cm}$ and the hub height at $10.5 \mathrm{~cm}$, occupied the lowest one-third of the boundary layer. High-resolution particle image velocimetry (PIV)
\end{abstract}

\footnotetext{
W. Zhang · C. D. Markfort

Saint Anthony Falls Laboratory, Department of Civil

Engineering, University of Minnesota,

Minneapolis, MN 55414, USA

e-mail: wzhang@umn.edu

C. D. Markfort

e-mail: mark0340@umn.edu

F. Porté-Agel $(\bowtie)$

Wind Engineering and Renewable Energy Laboratory (WIRE),

École Polytechnique Fédérale de Lausanne (EPFL),

ENAC-IIE-WIRE, 1015 Lausanne, Switzerland

e-mail: fernando.porte-agel@epfl.ch
}

was used to measure velocities in multiple vertical streamwise planes $(x-z)$ and vertical span-wise planes $(y-z)$. In particular, we identified localized regions of strong vorticity and swirling strength, which are the signature of helicoidal tip vortices. These vortices are most pronounced at the top-tip level and persist up to a distance of two to three rotor diameters downwind. The measurements also reveal strong flow rotation and a highly non-axisymmetric distribution of the mean flow and turbulence structure in the near wake. The results provide new insight into the physical mechanisms that govern the development of the near wake of a wind turbine immersed in a neutral boundary layer. They also serve as important data for the development and validation of numerical models.

\section{Introduction}

Turbulent wakes downwind of horizontal-axis wind turbines have been studied extensively through field observation, laboratory experiments and numerical simulations (Crespo et al. 1999). Knowledge of wake development behind the turbine rotors is important for the minimization of wake interference effects and the optimization of wind turbine performance in wind farms. As Whale et al. (1997) pointed out, the mean wake characteristics, their relation to the incident wind field and the local topography, influence the total energy resource at a potential wind farm site. Furthermore, the turbulence structure, and particularly the turbulence intensity distribution in the wake, affects the fatigue loading of downwind turbines. These factors should be considered for optimizing the layout of wind farms. The design of wind farms, therefore, can benefit significantly from detailed information about these fundamental wake characteristics. 
Aerodynamic research of wind turbines has contributed significantly to the success of modern wind energy. Interested readers are referred to a comprehensive review of wind-turbine-wake research conducted by Vermeer et al. (2003). Wind-tunnel experiments have been widely used to study wind turbine wakes (e.g., Whale et al. 2000; Vermeer 2001; Medici and Alfredsson 2006; Dobrev et al. 2008). These measurements have provided valuable insights into the aerodynamics of wind turbines, such as studies by NREL at NASA Ames (Simms et al. 2001) and the recent European-funded MEXICO wind-tunnel experiments which included detailed flow-field measurements in the wake of a $4.5 \mathrm{~m}$ diameter rotor (Snel et al. 2007; Sørensen 2011). Data collected under well-controlled conditions are particularly useful to validate the performance of numerical wake models. However, most previous laboratory studies were performed under uniform, freestream flow conditions (see review by Vermeer et al. 2003), which are not fully representative of the non-uniform characteristics of the atmospheric boundary layer where wind turbines operate. Only recently, wind-tunnel experiments have been designed and carried out with the purpose of understanding the effects of the incoming flow (free-stream vs. boundary layer over rough and smooth surfaces) and thermal stability (stable vs. neutral) on the wake structure by Chamorro and Porté-Agel (2009) and Chamorro and Porté-Agel (2010), respectively. Also, Hancock and Pascheke (2010) investigated the behavior of a stand-alone and multiple wind turbine wakes in a stable boundary layer wind tunnel. Cal et al. (2010) studied the vertical transport of kinetic energy from a neutral boundary layer flow to an array of model wind turbines using windtunnel experiments. These works highlight the importance of considering the effects of boundary layer flows on wind turbine wake structures.

Previous measurements behind turbine models have mainly been limited to measurements of the stream-wise or axial velocity component in order to determine the wake development and the velocity deficit. The velocity deficit in the far wake is usually assumed as axisymmetric and selfsimilar in analytical models (Burton et al. 2001). However, the near wake is characterized by a complex coupled vortex system (including helicoidal tip vortices), unsteadiness and strong turbulence heterogeneity. Limited information about the spatial distribution of turbulence in the near wake, the vortex behavior and their influence on the downwind development of the far wake hinders our capability to predict wind turbine power production and fatigue loads in wind farms. For instance, Hansen and Butterfield (1993) pointed out that knowledge of all three velocity components in the flow field is essential in order to predict rotor loads in a wind farm. Therefore, it is of great interest to understand the full velocity field, including all three velocity components and all components of the turbulent stress tensor in the near wake.

Turbulence intensity is used as a measure of the fatigue loads on various components of the turbine. Also, the power fluctuations of a wind turbine depend primarily on the turbulence intensity of the wind. Models for turbulence levels in turbine wakes do not consider cross-sectional variability. In many applications, it is of importance to estimate reliable turbulence stresses in the wake. For example, to calculate the unsteady loads induced by turbulence, it is critical to understand the spatial correlation structure of the turbulence in all three directions. To date, no accurate model exists for describing a turbulent wind field within the turbine wake, especially in the near-wake region.

The major source of three-dimensionality of the near wake is the complicated vortical system that forms immediately behind the rotor. Particle image velocimetry (PIV) measurements are able to yield the turbulence statistics of the wake flow and also reveal the detailed vortex structure of the wake by mapping the vorticity contours. One of the earliest applications of PIV to study wind turbine wakes is by Whale et al. (1997). They measured mean and turbulent wake characteristics at distances of 1.1 and 1.5 rotor diameters away from the model turbine in the near wake and compared their results with full-scale data collected in the field. Additionally, Whale et al. (2000) carried out a series of experiments to characterize tip vortex properties of a two-blade turbine in the free-stream flow in a water tank. Grant and Parkin (2000) also made PIV measurements of two- and three-blade wind turbines in order to track the tip vortices. Medici and Alfredsson (2006) studied the wind turbine wake under yaw conditions. Dobrev et al. (2008) presented quantitative information of the wake downstream of a model wind turbine. Most of the experiments mentioned previously were done with a free-stream inflow condition, without simulating an incoming boundary layer flow. As stated previously, it is essential to correctly simulate the boundary layer with high shear and high turbulence level, which significantly affects the wake structure (e.g., Chamorro and Porté-Agel 2009).

In this study, we report new wind-tunnel data collected in the near wake of a model wind turbine immersed in a neutrally stratified boundary layer. Previous work by Chamorro and Porté-Agel (2009) discussed the velocity deficit and enhanced turbulence in the far wake of the wind turbine in great detail. Our particular focus is on understanding and quantifying the development of the near wake by revealing the tip vortex properties and characterizing the spatial distribution of turbulence statistics, including mean velocities, turbulence intensities and Reynolds shear stresses. This study seeks to improve our understanding of the turbulent wake structure, especially in the complex near-wake region. 


\section{Experimental setup}

\subsection{Boundary layer wind tunnel}

Experiments were carried out in the recirculating boundary layer wind tunnel at the Saint Anthony Falls Laboratory of the University of Minnesota. The main test section, designed for atmospheric boundary layer studies, has a development length of $16 \mathrm{~m}$ and a cross-section of $1.7 \times 1.7 \mathrm{~m}^{2}$. A turbulent boundary layer was developed with the help of a tripping mechanism $(80 \mathrm{~mm}$ picket fence) at the entrance of the wind-tunnel test section. The test section floor is made up of a series of smooth aluminum plates for which temperature can be controlled by an automated heating and cooling system, to achieve the desired thermal stratification condition (neutral, stable or unstable). The range of controlled temperature of both the air and the floor is within $\pm 0.25^{\circ} \mathrm{C}$ between 5 and $80^{\circ} \mathrm{C}$. In this experiment, the temperature of the air $\left(T_{f}\right)$ and the floor $\left(T_{s}\right)$ were kept at a constant temperature of $30^{\circ} \mathrm{C}$ to simulate a neutral boundary layer in the test section. The freestream turbulence intensity is approximately $2 \%$ at $U_{f}=2.5 \mathrm{~m} \mathrm{~s}^{-1}$. More details about the wind tunnel can be found in Carper and Porté-Agel (2008).

\subsection{Measurement methodology}

Two measurement techniques were employed to acquire detailed flow information. A high-resolution stereoscopic
PIV or S-PIV system from TSI, Inc. was used to measure three velocity components in 2D planes from two orthogonal views: vertical stream-wise $(x-z)$ planes and vertical span-wise/cross-sectional $(y-z)$ planes. A dual-head Nd:Yag laser (Quantel-USA) was used as the light source which can produce a laser pulse of $190 \mathrm{~mJ}$. The laser beam was transferred by an optical arm to the wind-tunnel test section and transformed into a light sheet using cylindrical and spherical lenses. Two PowerView Plus 4MP 12-bit CCD cameras $(2,048 \times 2,048$ pixels $)$ fitted with $105 \mathrm{~mm}$ lenses were setup with Scheimpflug mountings to capture particle images in $x-z$ planes. In addition to S-PIV measurements, conventional 2-Dimensional, 2-Component (2D2C) PIV was also performed in multiple $y-z$ planes, at $x / d=1,2,3$ and 5, with the help of a reflection mirror located at eight rotor diameters downwind from the measurement plane. Figure 1 shows a schematic diagram of the measurement setup.

Olive oil particles, tracers for PIV measurements, were generated using a homemade atomizer. Six cylindrical reservoirs were filled half-full with olive oil, and compressed air was forced through Laskin nozzles to generate $1 \mu \mathrm{m}$ oil droplets which were released into the upper part of the chamber. The atomized olive oil droplets went into the entrance of the test section, where they were allowed to mix with the airflow sufficiently and fill homogeneously prior to measurements.

Calibration of the 2D2C PIV was accomplished by imaging a grid target placed in the laser light sheet. This
Fig. 1 Schematic of the experimental setup, coordinate system and measurement locations (top). Geometric parameters of the model wind turbine (bottom)

way a scale factor of the current PIV setup was obtained to convert the length scale from the measurement plane to the image plane. S-PIV calibrations followed the multi-plane procedure described by Raffel et al. (2007). Particle images were evaluated to determine instantaneous velocity fields using a multigrid method having second-order accuracy (Insight 3G program from TSI, Inc.). The final interrogation window of $32 \times 32$ pixels is analyzed with $50 \%$ interrogation window overlapping. Erroneous vectors, less than $1 \%$ of the total calculated vectors, were replaced by vectors interpolated through a Gaussian scheme from valid neighboring vectors. The field of view (FOV) and spatial resolution of representative PIV measurements are summarized in Table 1 . The mean velocity field was obtained by ensemble averaging 1,500 instantaneous velocity fields. All fluctuating velocity fields, derived by subtracting the mean velocity field from the instantaneous velocity fields, were used to compute turbulence intensities and Reynolds shear stresses. Because the flow is time dependent, these results represent a statistical average of the flow field for a large number of rotor revolutions. The measurement uncertainty level of the velocity vectors is estimated to be within $2 \%$ and approximately $6 \%$ for the vorticity using a central difference scheme.

In addition, a hot-wire anemometer (HWA) was used to measure instantaneous stream-wise and wall-normal velocity components with high temporal resolution at selected downwind locations. The probe is made of two $5 \mu \mathrm{m}$ tungsten wires, which are connected to an A. A. Lab Systems AN-1003 10-channel constant temperature anemometry (CTA) system. The sensor was mounted on a traversing system (Velmex, Inc) to measure vertical profiles at multiple locations. As shown in Fig. 1, the crosswire anemometer was positioned at selected downwind stream-wise locations in the central plane $(y=0)$ of the turbine wake $(x / d=2,3,5,10$, and 20). Data were collected in vertical profiles starting at $z=1 \mathrm{~cm}$ up to $z=40$ $\mathrm{cm}$ with intervals of $1 \mathrm{~cm}$ between measurement points. Data were sampled for 60-120 s at a frequency of 1,000 or $2,000 \mathrm{~Hz}$ depending on the specific stream-wise location.

The hot-wire anemometer was calibrated in a wellcontrolled small calibration unit against a Pitot-static

Table 1 Field of view and spatial resolution of PIV measurement cases

\begin{tabular}{llll}
\hline Measurement station & $\begin{array}{l}\text { Field of } \\
\text { view } \\
\left(\mathrm{mm}^{2}\right)\end{array}$ & $\begin{array}{l}\text { Spatial } \\
\text { resolution } \\
(\mathrm{mm})\end{array}$ & Vectors \\
\hline$x-z$ planes $(x / d=0-3)$ & $146.9 \times 135.4$ & 2.3 & $128 \times 118$ \\
$\begin{array}{l}y-z \text { planes }(x / d=1,2, \\
3,5)\end{array}$ & $149.8 \times 188.7$ & 3.3 & $92 \times 116$ \\
\hline
\end{tabular}

probe, at seven inclination angles and multiple wind velocities at $30 \pm 0.25^{\circ} \mathrm{C}$. A cubic-spline lookup table calibration method was then used to determine instantaneous velocities from the two instantaneous voltages. Calibration of the anemometer was performed before and after the experiments to verify the calibration throughout the entire experiment. During the calibration and measurements, the temperature fluctuations were held within $\pm 0.25^{\circ} \mathrm{C}$ to avoid bias errors due to thermal drift of the voltage signal. More details on the calibration procedure can be found in Bruun (1995). Velocities measured with the hot-wire can be considered accurate to at best $1 \%$. The accuracy of the turbulence intensity in a wall-bounded flow depends on a complex combination of probe length, wall-normal positioning and sensor Reynolds number (see Jorgensen 1996; Hutchins et al. 2009).

\subsection{Experimental conditions}

The experiments were conducted with a free-stream velocity of approximately $2.5 \mathrm{~m} \mathrm{~s}^{-1}$. A boundary layer was allowed to grow to $\delta \approx 0.6 \mathrm{~m}$ at the measurement location. The friction Reynolds number of this neutrally stratified boundary layer (based on the friction velocity of $u_{*}=0.12 \mathrm{~m} \mathrm{~s}^{-1}$ ) is $R e_{v}=u_{*} \delta / v \approx 4.6 \times 10^{3}$. The aerodynamic surface roughness length $z_{0}$ was estimated to be $0.021 \mathrm{~mm}$ from the log-law profile using averaged streamwise velocity in the surface layer (approximately lowest $10-15 \%$ of the boundary layer). The normalized standard deviation of the stream-wise and vertical velocity components near the surface is $\sqrt{\overline{u^{\prime 2}}} / u_{*}=2.47-2.25$ and $\sqrt{\overline{w^{\prime 2}}} / u_{*}=1.08-1.14$, falling into the typical value range of the surface layer values in a neutrally stratified atmospheric boundary layer simulated in wind tunnels: $\sqrt{\overline{u^{\prime 2}}} / u_{*}=2.1-2.5$ and $\sqrt{\overline{w^{\prime 2}}} / u_{*}=1.0-1.2$ (e.g., Grant 1992; Gong and Ibbetson 2009; Athanassiadou and Castro 2001; Khurshudyan et al. 1981). Figure 2 shows the vertical profiles of the mean stream-wise and vertical velocities and turbulence intensities measured in the boundary layer normalized by the rotor diameter $d$ and the incoming mean velocity measured at the rotor hub height ( $\left.U_{\text {hub }}\right)$.

The miniature wind turbine model is designed in a way that the key geometric parameters are comparable to those of large-scale wind turbines in the field. There is only a limited number of wind-tunnel experiments designed to study the wake of rotating wind turbine models. Most experiments involve the use of stationary models (e.g., porous disks), as summarized in Vermeer et al. (2003) and Medici and Alfredsson (2006). The model wind turbine employed here is a functional turbine that consisted of a three-blade GWS/EP-5030 $\times 3$ rotor driving a small DC 

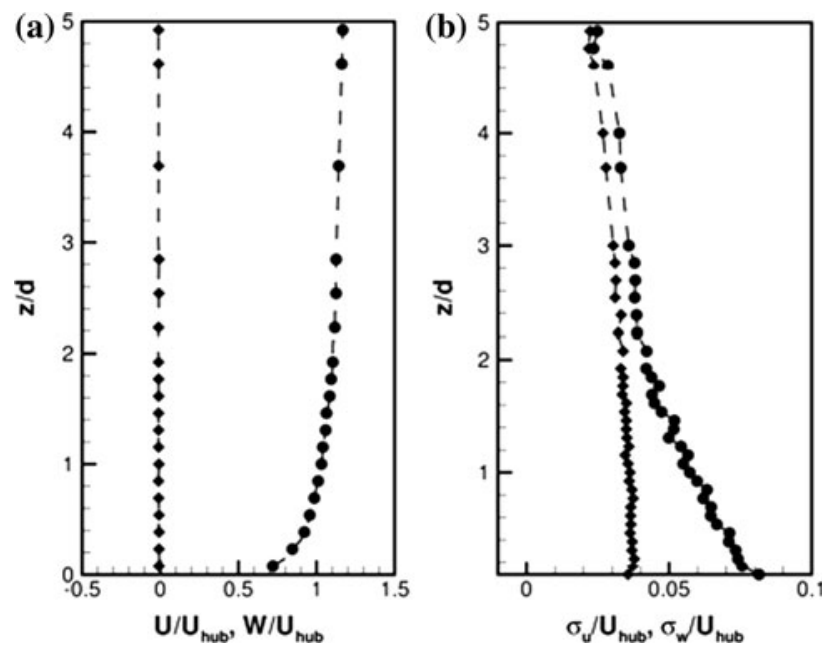

Fig. 2 Characteristics of the NBL simulated over a smooth surface: a Normalized mean stream-wise (circle) and vertical (diamond) velocities and b Stream-wise (circle) and vertical (diamond) turbulence intensities. Height above the surface $z$ is normalized with the rotor diameter $d$

generator to extract energy from the wind. The blades of this rotor are nearly flat plates, similar to those of the small homemade wind turbine models used in Lebrón et al. (2010) and Cal et al. (2010). The tower is $10.5 \mathrm{~cm}$ and the rotor diameter $d$ is $13 \mathrm{~cm}$, with the bottom tip at $4 \mathrm{~cm}$ above the ground and the top tip at $17 \mathrm{~cm}$. As shown in Fig. 1, the bottom tip of the turbine is set at the height about 0.6 times the turbine radius, which is similar to that of large-scale turbines; and the turbine rotor plane is within the lowest one-third of the boundary layer.

The rotor operates at $1,140-1,160 \mathrm{rpm}$ with a freestream velocity of $2.5 \mathrm{~m} \mathrm{~s}^{-1}$, giving a tip speed ratio $\lambda\left(=\Omega(2 \pi / 60)(d / 2) / U_{\text {hub }}\right.$, that is, ratio of the tangential tip velocity to the undisturbed wind speed measured at the hub height) of approximately 3.7. This tip speed ratio falls into the range of $\lambda$ for typical three-blade wind turbine models used in wind-tunnel tests, from 3 to 6.7 (Vermeer et al. 2003). Note that full-scale wind turbines operate in a wider range of tip speed ratios, with the optimum values around $6-8$. For the conditions of this experiment $(\lambda=3.7)$, the thrust coefficient $C_{T}$ and the power coefficient $C_{p}$ are estimated to be 0.42 and 0.37 . The coefficients are close to those of wind turbine models used in previous wind-tunnel experiments, as listed in Table 2.

In general, the Reynolds number of the atmospheric flow cannot be matched by wind-tunnel experiments. The Reynolds number in this study is lower than that of a fullscale wind turbine flow by a factor of about 100. Even though the influence of Reynolds number on turbine wakes is not fully understood, previous studies have shown that primary wake characteristics such as wake rotation and tip vortices can be reproduced even at relatively low Reynolds
Table 2 Operational parameters of three-blade wind turbine models used in wind-tunnel studies

\begin{tabular}{llll}
\hline Studies & $\begin{array}{l}\text { Tip speed } \\
\text { ratio, } \lambda\end{array}$ & $\begin{array}{l}\text { Power } \\
\text { coefficient, } C_{p}\end{array}$ & $\begin{array}{l}\text { Thrust } \\
\text { coefficient, } C_{T}\end{array}$ \\
\hline The present study & 3.7 & 0.37 & 0.42 \\
$\begin{array}{c}\text { Medici and } \\
\quad \text { Alfredsson (2008) }\end{array}$ & $0.42-4.87$ & $0-0.32$ & $0.13-0.35$ \\
$\begin{array}{c}\text { Cal et al. (2010) } \\
\text { Hancock and }\end{array}$ & 4.9 & 0.29 & 0.32 \\
$\quad$ Pascheke (2010) & 6.05 & 0.293 & 0.497 \\
\hline
\end{tabular}

number (Medici and Alfredsson 2006). This highlights the value of wind turbine wake data acquired in wind tunnels for the purpose of understanding the fluid dynamics. Furthermore, as long as the aerodynamic characteristics of the rotor blades are known in the modeled Reynolds number range, wind-tunnel data of turbine wakes provide valuable benchmarks for comparison with and validation of numerical models (Vermeer et al. 2003).

Throughout this paper, we use the following definitions: the wind velocities $U, V, W$ are mean wind speeds in the stream-wise $(x)$, lateral/span-wise $(y)$ and vertical (z) directions, and $u^{\prime}, v^{\prime}$ and $w^{\prime}$ are deviations of the instantaneous wind speed components $(u, v$, and $w)$ from the mean wind speed.

\section{Results and discussion}

\subsection{Signature of tip vortices}

Tip vortices are distinct features of horizontal-axis wind turbine wakes; they are shed from the tip of each blade and transported downwind and eventually dissipated. Observed both in free-stream flow and boundary layer flow, tip vortices follow a helical path and dissipate faster in the latter than in the former conditions (Pedersen and Antoniou 1989; Porté-Agel et al. 2011). In order to identify coherent vortex structures, we first used PIV data to map the mean and instantaneous fields of vorticity and swirling strength.

Contours of the ensemble-averaged stream-wise vorticity $\left(\omega_{x}=0.5(\partial v / \partial z-\partial w / \partial y)\right)$ calculated using PIV data in the vertical span-wise $(y-z)$ planes are in Fig. 3. The stream-wise vorticity contours show a "double ring" pattern at $x / d=1$. The intensive vorticity can be interpreted as the signature of the helicoidal tip vortices advected through the measurement plane and the shear layers formed on the edge of the wake. In particular, the magnitude of vorticity is noticeably higher at the edge of the upper half of the rotor-swept area, owing to the non-uniformity of the incoming boundary layer flow. In addition, at the center of the rotor-swept area, strong negative vorticity is generated 
Fig. 3 Contours of the ensemble-averaged stream-wise vorticity $\left(\omega_{x}\right)$ in the span-wise planes of the near wake. $\mathbf{a} x / d=1 ; \mathbf{b} x / d=2 ; \mathbf{c} x / d=3$ and $\mathbf{d} x / d=5$. The dashed line indicates the turbine rotor plane
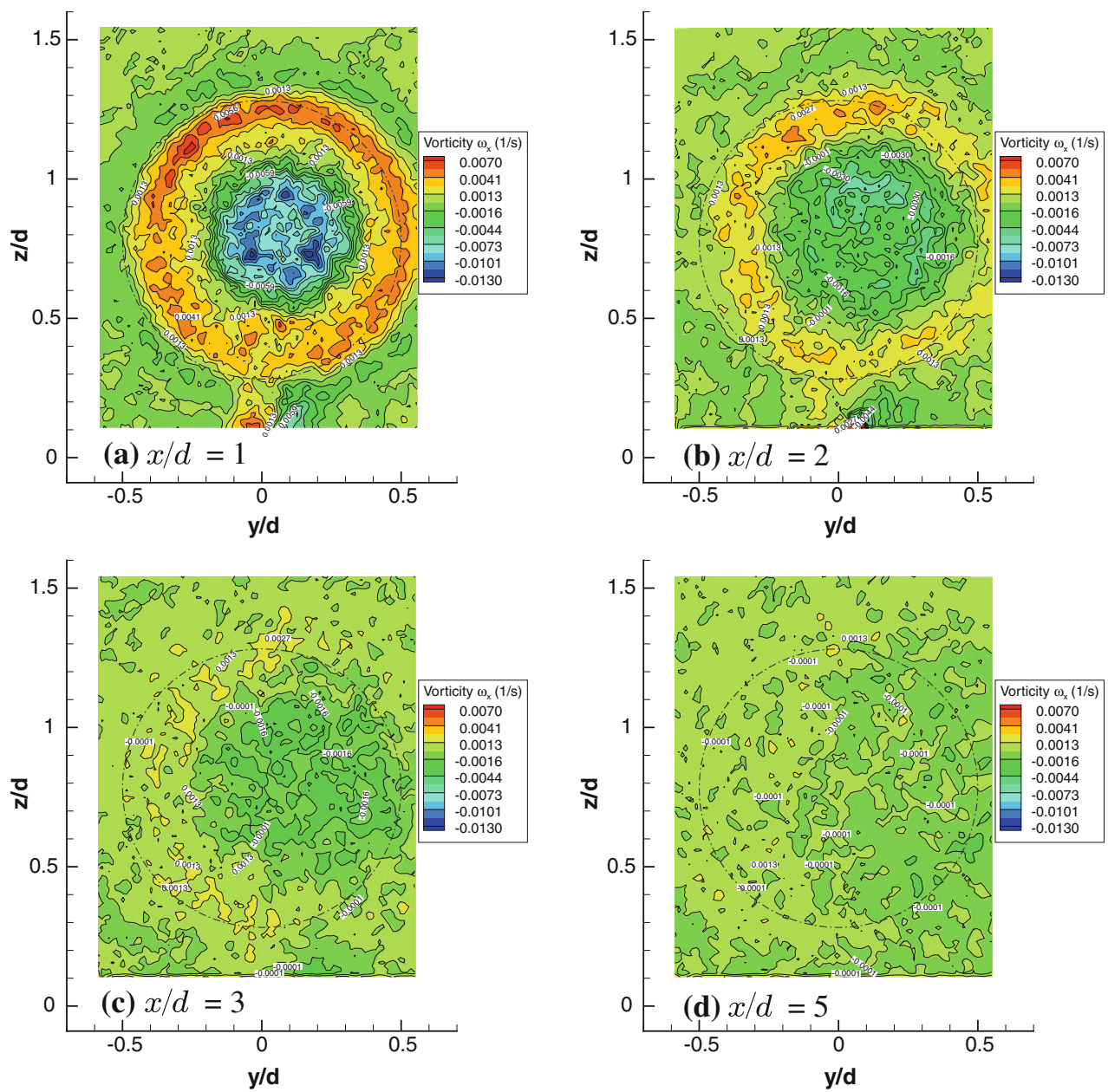

around the hub, which is the signature of the hub/root vortices. As expected, the intensive vorticity expands and diffuses with downwind distance, indicative of wake expansion. At $x / d=5$, the vorticity distribution becomes nearly homogeneous over the entire rotor-swept area, and the magnitude of $\omega_{x}$ is higher than that of the incoming flow.

Since concentrated vorticity can be generated by either coherent vortices or shear layers, swirling strength $\left(\lambda_{c i}\right)$ (Adrian et al. 2000; Zhou et al. 1999) was used to improve the identification of vortex cores. Defined as the imaginary part of the complex eigenvalues of the complex velocity gradient tensor, the swirling strength is used to differentiate spiraling motion around an axis normal to the 2D measured plane from rotation caused by a shear layer. Figure 4 shows the color-coded span-wise vorticity $\left(\omega_{y}=0.5(\partial w / \partial x-\right.$ $\partial u / \partial z)$ ) distribution overlaid with swirling strength (red lines) in the central wake plane at $x / d=0-3$. The distribution of swirling strength is well matched with vorticity. Strong positive and negative vorticities in the upper and lower part of the wake immediately behind the nacelle indicate alternating vortex shedding. A band of intensive

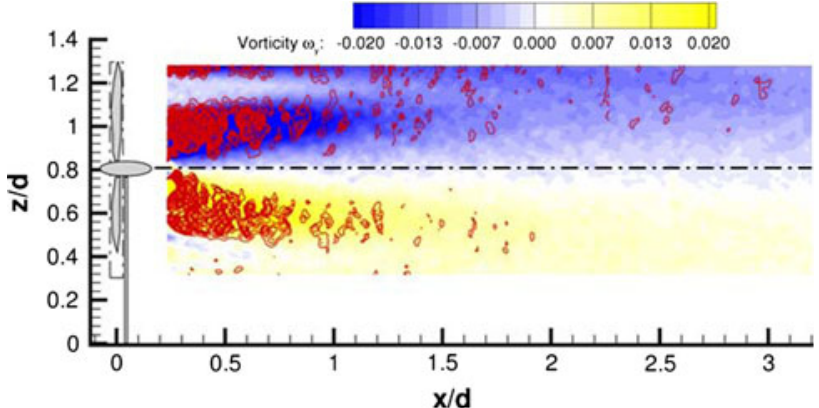

Fig. 4 Contours of the ensemble-averaged span-wise vorticity $\left(\omega_{y}\right)$ overlaid with swirling strength $\left(\lambda_{c i}\right)$ distribution in the center plane of the wake $(y=0, x / d=0-3)$. The contours of swirling strength are presented using red lines. The horizontal dashed line indicates the turbine hub height

vorticity as well as swirling strength contours is found at the top-tip height, but not at the bottom-tip level. This is due to the fact that tip vortices dissipate slower and thus persist over a longer distance at the top-tip height, where the incoming flow has a relatively low turbulence level compared to a higher turbulence level at the bottom-tip 
height. The hub/root vortices are significant prior to $x / d=1.5$ and then rapidly dissipate beyond this distance. The top-tip vortices are still visible as far as $x / d=2$. Lu and Porté-Agel (2011) also found top-tip vortices persist until about two rotor diameters downwind of the turbine, in their high-resolution large-eddy simulations (LES, with an actuator line model) of the wake behind a wind turbine immersed in a stable boundary layer.

Top-tip vortices can also be identified in the instantaneous velocity fields immediately downwind of the turbine $(x / d=0-1)$, as shown in Fig. 5. Four or five tip vortices are visible at the top-tip height, but not at the bottom-tip height. Reviewing the series of instantaneous velocity fields, we notice that tip vortices occur consistently and not randomly. Qualitatively the tip vortices observed here are very similar to those reported in Dobrev et al. (2008).

The present PIV data reveals a complicated vortex system, composed of tip vortices and hub/root vortices in the near-wake downwind of a model wind turbine. The hub/root vortices are significant prior to $x / d=1.5$ and dissipate fast. The top-tip vortices remain until $x / d=2$. Previous work to visualize tip vortices shed from the wind turbine blades using PIV was reported in Dobrev et al. (2008), Grant et al. (1997), Grant and Parkin (2000) and Sherry et al. (2010). Among them, Whale et al. (2000) and Sherry et al. (2010) also revealed the hub/root vortices immediately behind the hub. In a low turbulence flow or in the free-stream, previous measurements show that the tip vortices are present beyond a distance of three rotor diameters, while the vortices behind the hub dissipate faster. One should note that the relative strength of hub/root vortices versus tip vortices observed in a wind tunnel may not be characteristic of field scale flows due to the ratio of hub to rotor blade size of a wind turbine. This ratio for wind turbines in the field is much smaller than that of the model wind turbine used in the present and most wind-tunnel studies, which suggests that relatively weaker hub/root vortices may occur in the field.
The period of tip vortex shedding can be estimated with the distance between two tip vortices $(\approx 0.25 d)$ and the stream-wise advection velocity $\left(1.8 \mathrm{~m} \mathrm{~s}^{-1}\right)$ at the top-tip height in instantaneous velocity fields (see Fig. 5). Using this approach, the shedding frequency of top-tip vortices is estimated to be approximately $55 \mathrm{~Hz}$. Additionally, spectral analysis was performed to examine the distribution of energy across a range of frequencies in the wake. The spectra were calculated by taking the fast Fourier transform (FFT) of the velocity fluctuations ( $u^{\prime}$ and $w^{\prime}, 2^{16}$ samples) utilizing a square window of $2^{12}$ points. These velocity fluctuations were measured by HWA at $1,000 \mathrm{~Hz}$ for $120 \mathrm{~s}$. The analysis shows the classical production subrange and inertial subrange for the surface layer turbulence, identified as regions that follow power law scaling with -1 and $-5 / 3$ slope, respectively (Katul et al. 1995). There are also localized high-energy signatures at a frequency corresponding to periodic coherent structures, that is, the tip vortices and large-scale wake meandering motions.

A concentration of turbulent kinetic energy is observed at frequencies of $55 \mathrm{~Hz}\left(3 f_{t}\right), 110 \mathrm{~Hz}\left(6 f_{t}\right)$ and $165 \mathrm{~Hz}\left(9 f_{t}\right)$, which are multiples of the frequency of consecutive rotating blades $3 f_{t}\left(f_{t}\right.$ is the rotation frequency of the turbine). Specifically, at $x / d=0.5,1.0$ and 1.5 , a distinct primary peak associated with the presence of tip vortices is observed at the top-tip level (Fig. 6). The primary peak is at a frequency of $3 f_{t}$ and the first and second harmonic frequencies are seen at $x / d=0.5$. The number of observed harmonic frequencies decreases with increasing distance from the turbine. At $x / d=1$, the primary and the first harmonic frequency can be seen while only the primary frequency remains at $x / d=1.5$ and 2 . Note that the frequency peak is not observed at the bottom-tip level (Fig. 6f), where the turbulence level of the incoming flow is relatively higher than that at the top-tip height and the wake interacts with the ground. This observation agrees with the PIV results presented previously.
Fig. 5 Tip vortex signature in instantaneous velocity fields at $x / d=0-1 ;$ a Snapshot 1 ; b Snapshot 2. The contours of swirling strength are presented using red lines
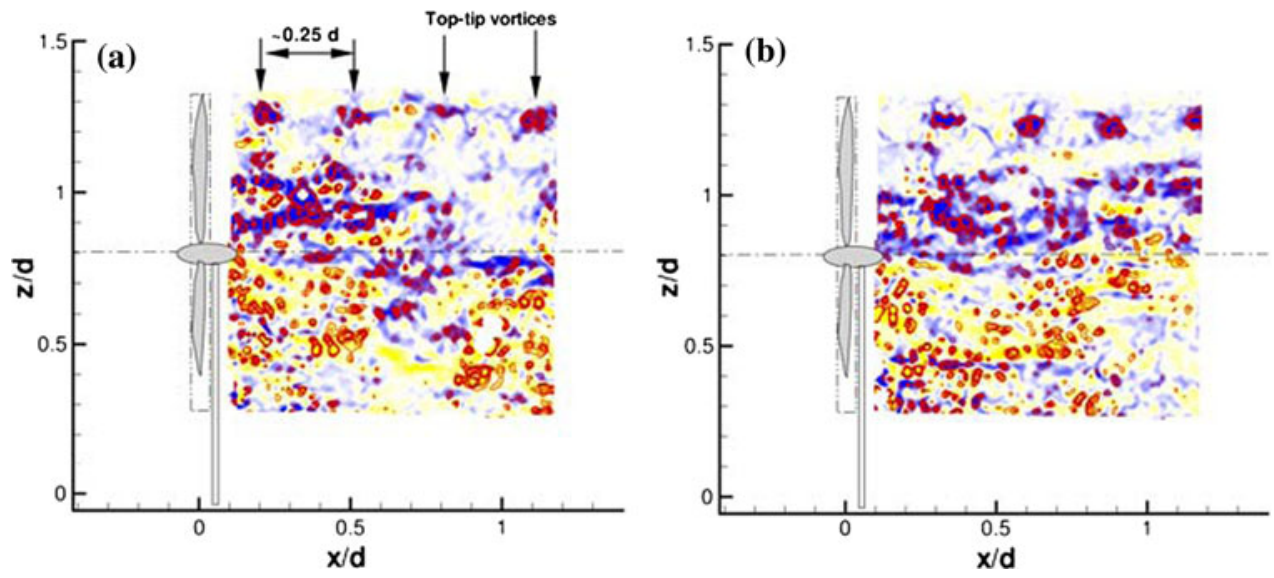

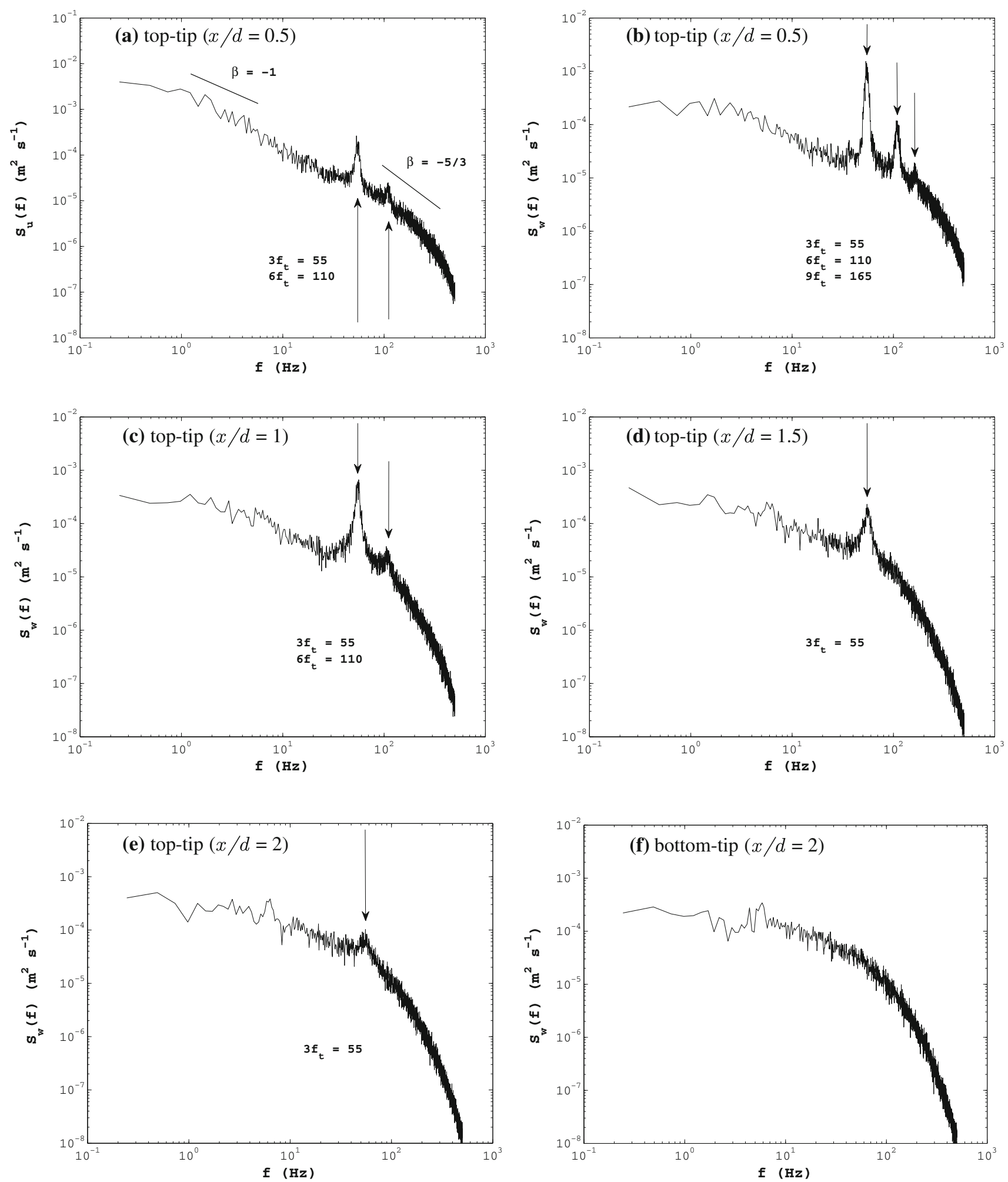

Fig. 6 Power spectrum of the velocity fluctuations $\left(u^{\prime}\right.$ and $\left.w^{\prime}\right)$ at the top-tip and bottom-tip heights in the center plane $(y=0)$ of the wake at selected downwind locations. a $S_{u}(f)$ at $x / d=0.5$, b $S_{w}(f)$ at

We also investigated the strength of the tip vortices in the side-tip planes. The power spectra of $u^{\prime}$ at the hub height and $0.5 d$, downwind of the turbine are examined in

$x / d=0.5$, c $S_{w}(f)$ at $x / d=1, \mathbf{d} S_{w}(f)$ at $x / d=1.5$, e $S_{w}(f)$ at $x / d=2$ (top-tip) and $\mathbf{f} S_{w}(f)$ at $x / d=2$ (bottom-tip)

Fig. 7. The spectra show stronger tip vortex signature at the near-tip side $(y / d=-0.5)$ compared to the far-tip side $(y / d=0.5)$. This is due to the fact that the wake rotates in a 

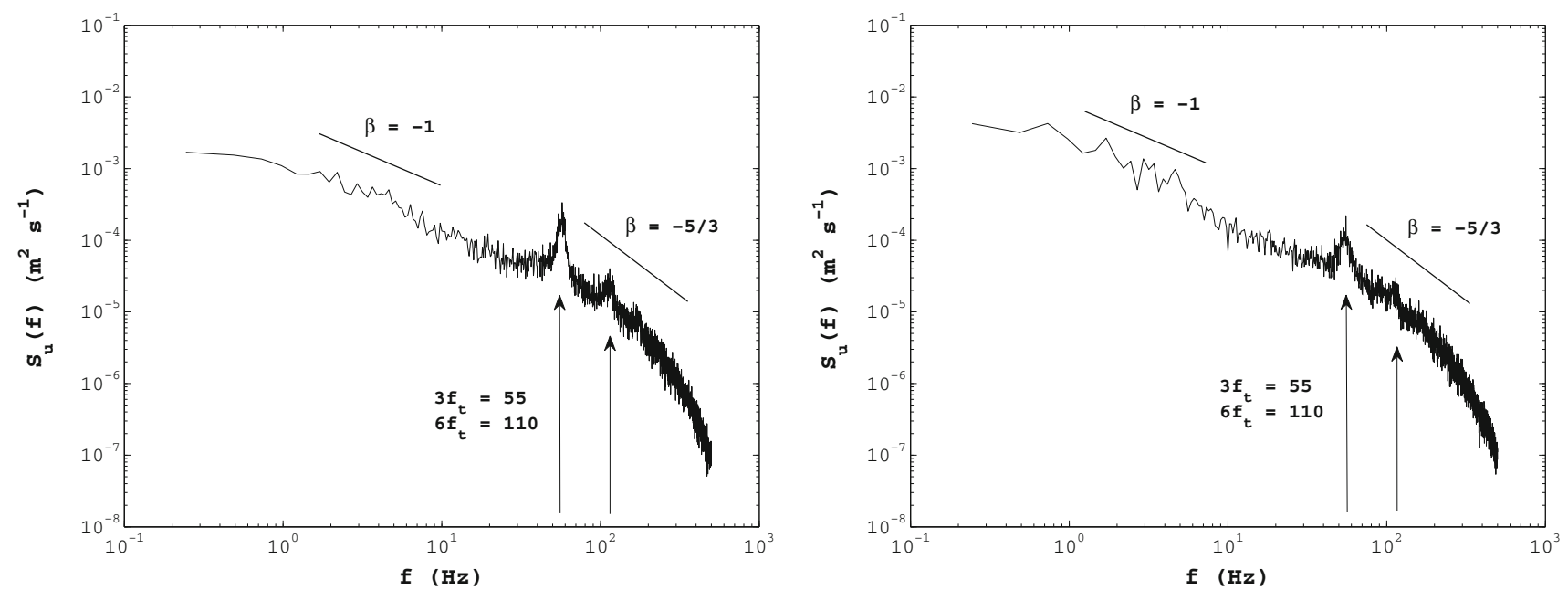

Fig. 7 Power spectrum of the stream-wise velocity fluctuation $\left(u^{\prime}\right)$ at the hub height and $0.5 d$ downwind of the turbine. The near-tip side $y / d=-0.5($ left $)$ and the far-tip side $y / d=0.5$ (right)

counterclockwise direction (viewed from the downwind of the turbine), thus bringing helicoidal vortices from below (where the background turbulence level is higher and the wake interacts with the ground) at $y / d=0.5$ and from above (where the background turbulence is weaker) at $y / d=-0.5$.

The power spectra of $w^{\prime}$ at the hub height in the center plane $(y=0)$ of the wake shows a strong low frequency peak at about $6 \mathrm{~Hz}$ at both $x / d=1$ and 2 (Fig. 8). This is consistent with previous work by Medici and Alfredsson (2006), who found a spectral peak at a lower frequency ( $\approx 8 \mathrm{~Hz}$ ) compared to the tip vortex shedding frequency, and attributed it to "meandering" of the whole wake. The resulting Strouhal number (calculated with the turbine rotor diameter $d$ and the local free-stream velocity) in their cases is between 0.12 and 0.2 and in our case about 0.35 . We, therefore, conclude that the low frequency motion detected here may be the overall effect of large-scale meandering of the wake structure. It should be noted that this self-induced instability is different from the wake meandering reported in field studies. In the field, large-scale atmospheric structures, which are comparable in size to the wake, are the main contributors to wake meandering (Bingöl et al. 2007; Larson et al. 2008). This wake meandering is correlated with the variability in the wind direction, resulting in "smoothed" or more homogeneous velocity profiles (Ainslie 1988; Whale et al. 1997). The type of bluff-bodyinduced wake meandering observed here has not been reported in field experiments, where near-wake data are scarce. It is also worthwhile mentioning that the ratio of the
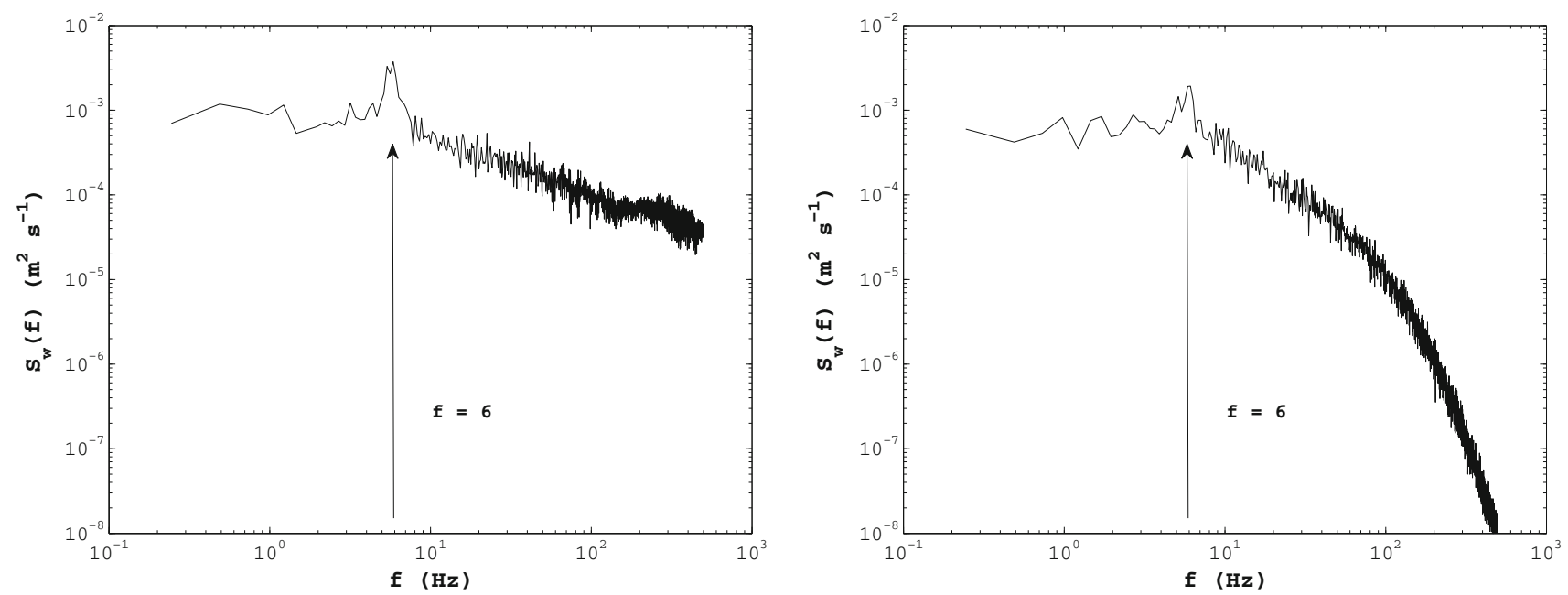

Fig. 8 Power spectrum of the vertical velocity fluctuation $\left(w^{\prime}\right)$ at the hub height in the center plane $(y=0)$ of the wake. $x / d=1(l e f t)$ and $x / d=2($ right $)$ 
nacelle size to the rotor diameter of wind turbines is much smaller in the field, which might make the self-induced instability effect not as prominent as in the wind tunnel.

\subsection{Mean wake characteristics}

The near-wake region is characterized by three-dimensional heterogeneously distributed turbulent flow that results from the complex interaction between the incoming boundary layer flow and the turbine-induced forces. These forces are responsible for the reduction in the axial velocity as well as the resulting rotation in the near-wake region. They also produce coherent vortex structures behind the turbine as described earlier. Highly three-dimensional flow structure is typically observed in the near wake of either stand-alone wind turbines (e.g., Wu and Porté-Agel 2011) or turbines in wind farms (see Porté-Agel et al. 2011; Lu and Porté-Agel 2011). In the following, we present the spatial distribution of the three velocity components measured in multiple vertical stream-wise $(x-z)$ and vertical span-wise $(y-z)$ planes in the near wake.

As mean kinetic energy is extracted from the wind by the wind turbine, there is a reduction in wind speed behind the rotor. Figure 9 shows an overall view of the mean wake structure in terms of three velocity component contours covering $x / d=0-3$ and $z / d=0.3-1.3$ (rotor-swept area) in the central wake plane $(y=0)$. A low momentum zone appears with nearly zero stream-wise velocity immediately downwind of the rotor $(x / d \leq 0.5)$. Flow reversal behind the nacelle and blade roots is observed in the region of $x / d \leq 0.4$. The flow reversal zone behind the nacelle is fairly large compared to the nacelle size, which indicates that it may be caused by both the nacelle and the root vortices. A large pressure gradient induced by strong nearwake rotation may also contribute to the amplification of the flow reversal zone. This complicated flow region, immediately downwind of the rotor, is strongly related to the rotor characteristics, such as the nacelle size, rotor blade geometry and hub-blade configuration. Expansion of the flow reversal zone was also reported by Massouh and Dobrev (2007), who explored detailed 3D flow properties behind a model wind turbine. Note that there is a lack of near-wake data $(x / d \leq 1)$ in the field measurements, hence it is not clear if this flow reversal exists behind the fullscale nacelle or not. Beyond this zone, the stream-wise velocity gradually increases and the wake continues to recover downwind. With respect to the rotor axis, the vertical distribution of stream-wise velocity is non-symmetric in this neutral boundary layer flow. The non-symmetric distribution of the stream-wise velocity extends throughout the wake region. Subtraction of the incoming flow results in the stream-wise velocity deficit, which is nearly symmetric with respect to the rotor axis in the far
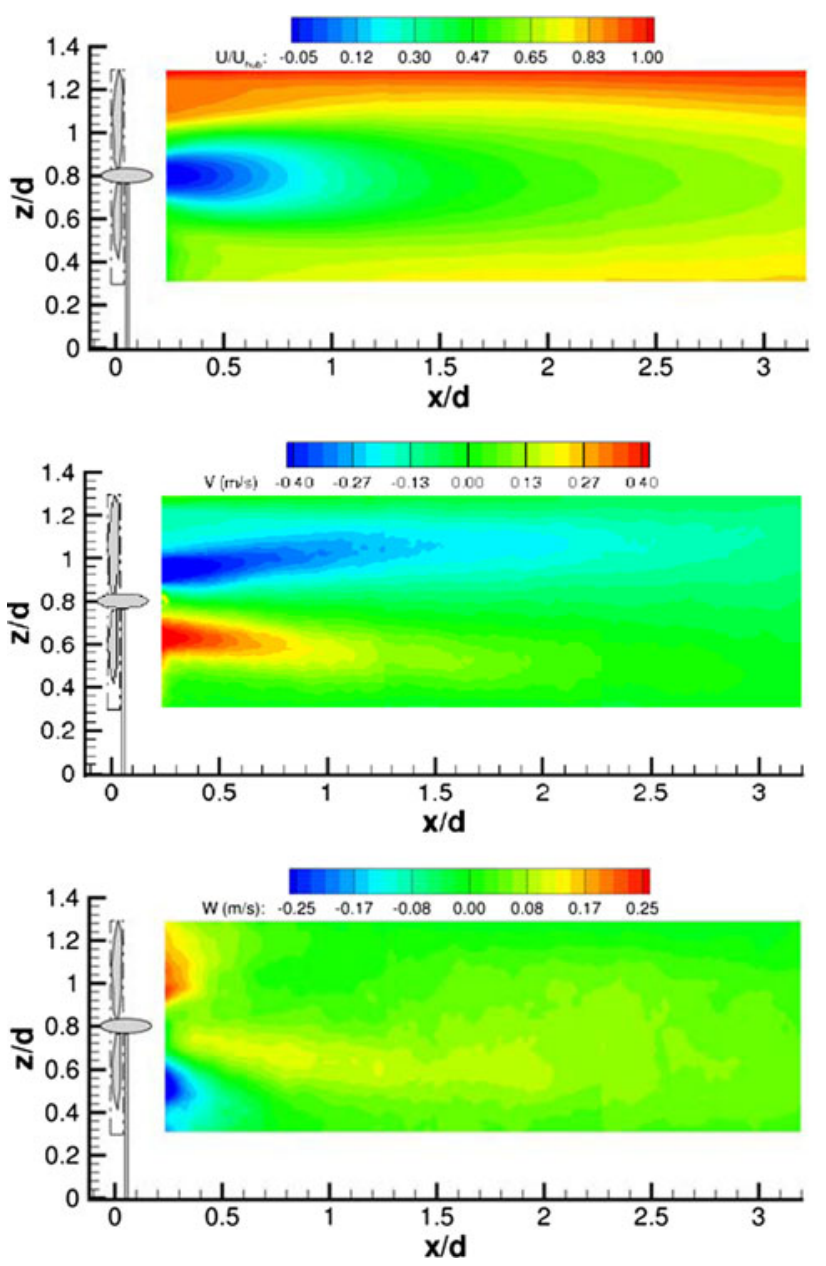

Fig. 9 Contours of mean velocities at $x / d=0-3$. The stream-wise velocity $U$ (top) is normalized by $U_{\text {hub }}$, while the vertical velocity $W$ (bottom) and the lateral velocity $V($ mid $)$ are not normalized

wake as discussed by Chamorro and Porté-Agel (2009). This facilitates modeling the velocity deficit in the far wake as axisymmetric and self-similar.

To capture the span-wise variation of the mean streamwise velocity $(U)$, contours of the stream-wise velocity are plotted in the center and two side-tip vertical planes in Fig. 10. Compared with the near-zero velocity zone in the central wake plane $(y=0)$, the stream-wise velocity in the two side-tip planes is much higher. However, the velocity or momentum deficit is still visible at the hub height and further downwind. The stream-wise velocity has higher values in the far-tip plane $(y / d=0.5)$ than the near-tip plane $(y / d=-0.5)$, which is attributed to the effect of wake rotation. This non-symmetric span-wise distribution of $U$ was also observed from previous wind-tunnel data in a vertical cross-sectional plane at $x / d=5$, presented by $\mathrm{Wu}$ and Porté-Agel (2011). It should be noted that the spanwise variation of the stream-wise velocity distribution still exists in the far wake $(x / d=5)$, where the flow rotation is weak. 


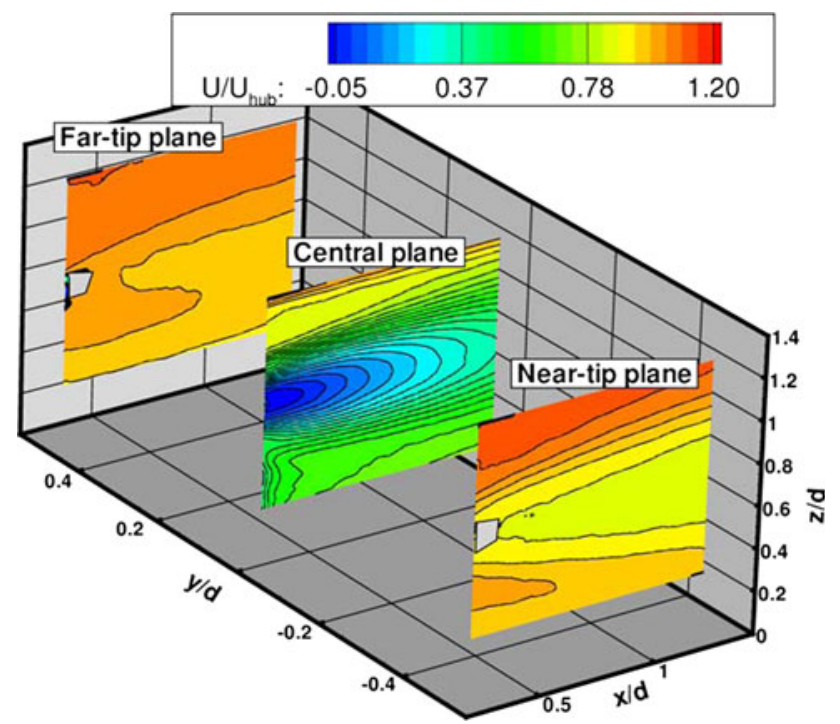

Fig. 10 Contours of the stream-wise velocity distribution in the central and two side-tip planes immediately downwind of the wind turbine. $(x / d=0.2-1.2, y / d=0$ and \pm 0.5$)$
The wake of a wind turbine is complicated by the fact that the rotor is composed of a finite number of blades that can independently affect the flow mainly through shedding vorticity (Vermeer et al. 2003). Moreover, due to conservation of angular momentum, the torque exerted by the wind on the rotor blades, which produces their rotation (clockwise, viewed from downwind of the turbine), leads to flow rotation in the opposite (counterclockwise) direction. The lateral velocity component $(V)$ shows rotation of the wake, with the positive into and the negative out of the $x-z$ plane in Fig. 9b. It is noticeable that the negative velocity contours persist longer compared to the positive contours in the lower part of the wake. This trend is again confirmed by the velocity fields captured in vertical spanwise planes (Fig. 11a, b). The maximum lateral velocity is about $0.4 \mathrm{~m} \mathrm{~s}^{-1}$ (20\% of the stream-wise velocity magnitude) and occurs close to the wind turbine. With increasing downwind distance, the lateral velocity decreases due to the turbulent mixing as the wake recovers. At $x / d=5$, wake rotation is not noticeable in the mean velocity field.
Fig. 11 Lateral velocity contours in span-wise planes at selected downwind locations. $\mathbf{a} x / d=1 ; \mathbf{b} x / d=2 ; \mathbf{c} x / d=3$; and $\mathbf{d} x / d=5$
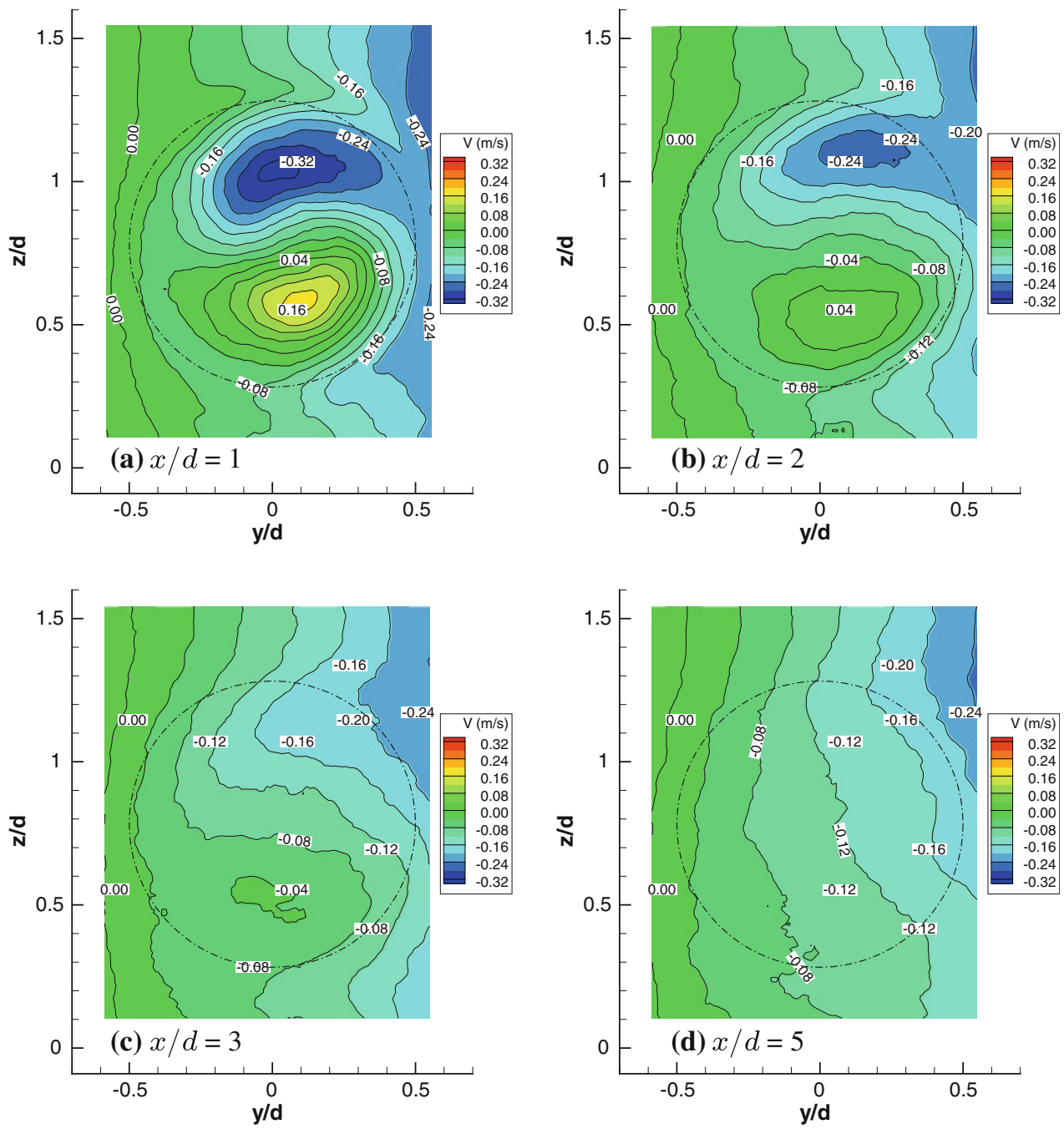
Similarly, the vertical velocity distribution also indicates wake rotation in both $x-z$ (in Fig. 9c) and $y-z$ planes. Contours of the average vertical velocity at the vertical cross-section planes and downwind location $x / d=5$ are shown in Fig. 12. The results show the non-symmetry (with respect to the hub height) of the velocity distribution owing to the incoming boundary layer flow and the presence of the ground surface. The maximum vertical velocity is approximately $0.24 \mathrm{~m} \mathrm{~s}^{-1}$, which is $75 \%$ of the maximum of the lateral velocity magnitude at $x / d=1$.

In summary, due to the effect of the wind turbine, the stream-wise velocity is reduced and the lateral and vertical velocities increase close to the rotor. With increasing distance to the rotor, the stream-wise velocity increases and the other velocities reduce. From the velocity field measured in the multiple vertical stream-wise and vertical span-wise planes, one can see that the near-wake region is characterized by high three-dimensionality and strong flow rotation. Recent LES studies capture the same flow characteristics and have shown that accounting for the non-uniform distribution of the turbine-induced forces and including rotation effects (using blade-element momentum theory) yields improved predictions of wind turbine wakes with respect to standard actuator disk models, especially in the near-wake region ( $\mathrm{Wu}$ and Porté-Agel 2011; Porté-Agel et al. 2011).

\subsection{Turbulent wake characteristics}

The ensemble-averaged PIV data clearly indicates that the maximum of the vertical and lateral velocity components, approximately $10-20 \%$ of the magnitude of the streamwise velocity, may not be neglected in the near wake. It is also important to understand the spatial distribution of other turbulence statistics, such as turbulence intensities and Reynolds shear stresses, in the near wake. Previous studies of wind turbine wakes found that the turbulence recovered more slowly than the mean velocity in the wind turbine wakes (Vermeer et al. 2003).

Figure 13 shows the high magnitude of the stream-wise turbulence intensity $\left(I_{u}=\sigma_{u} / U_{\mathrm{hub}}\right)$ within the rotor-swept
Fig. 12 Vertical velocity contours in span-wise planes at selected downwind locations. $\mathbf{a} x / d=1 ; \mathbf{b} x / d=2 ; \mathbf{c} x / d=3$; and $\mathbf{d} x / d=5$
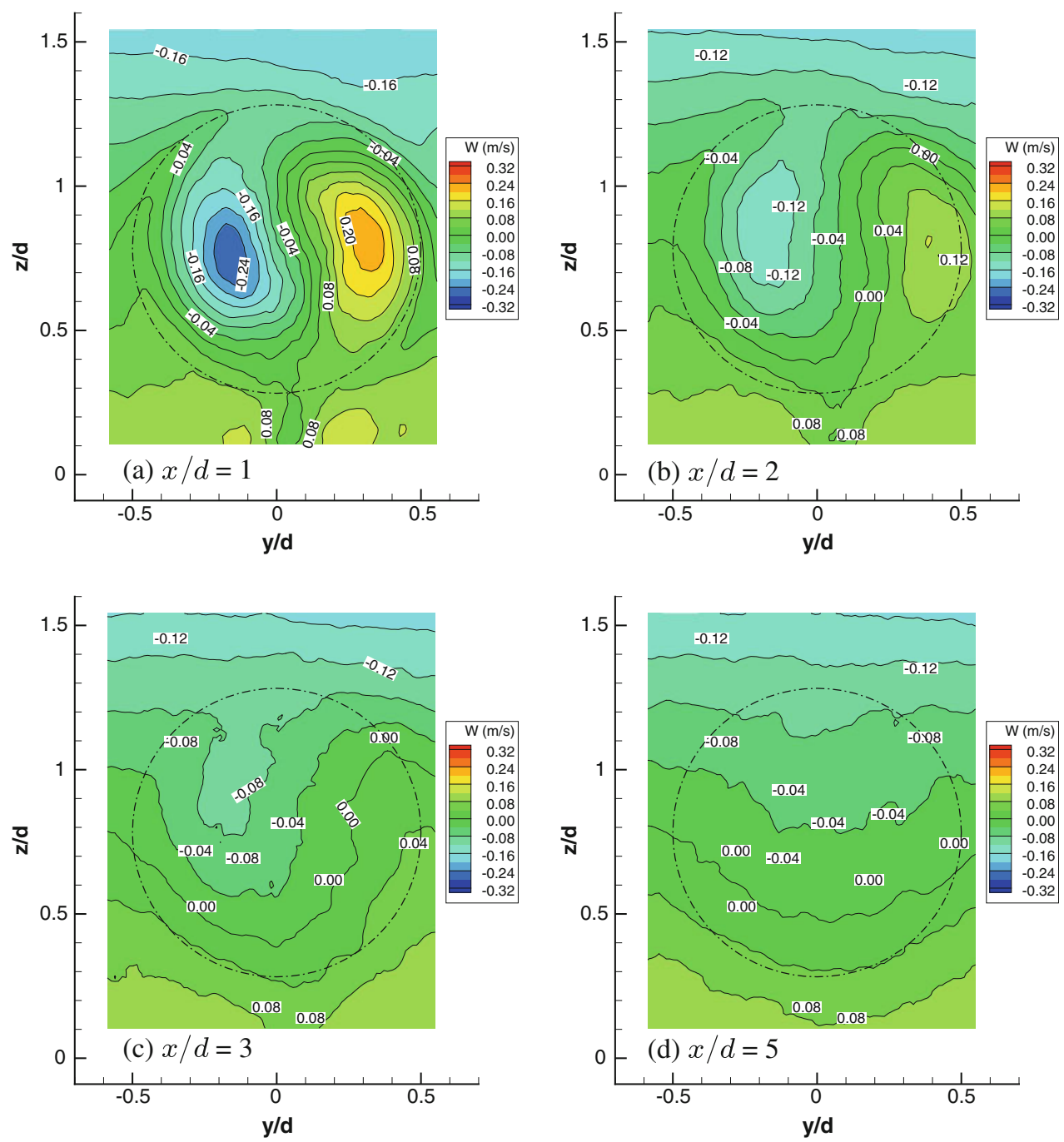

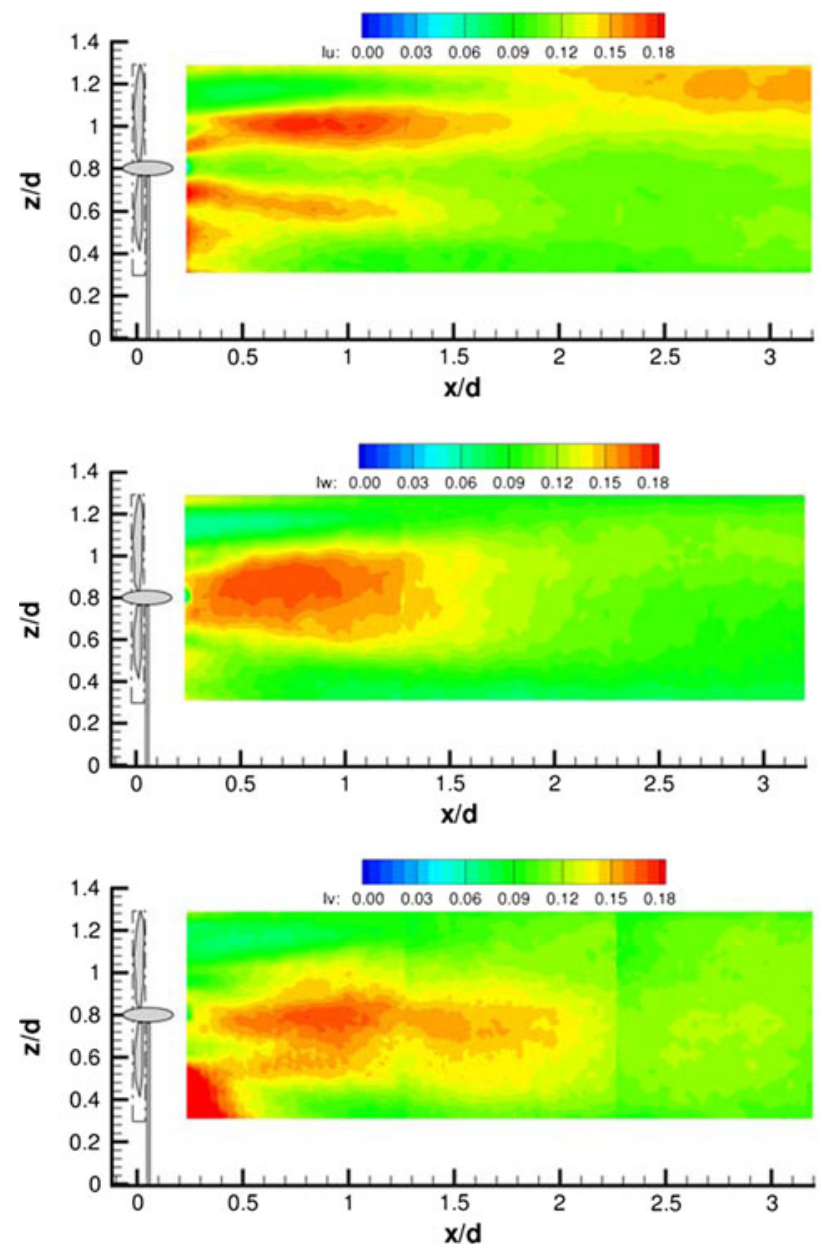

Fig. 13 Turbulence intensity contours in the near-wake region $(x / d=$ $0-3$ ). The stream-wise turbulence intensity $I_{u}=\sigma_{u} / U_{\text {hub }}$ (top), vertical turbulence intensity $I_{w}=\sigma_{w} / U_{\text {hub }}$ (mid) and lateral turbulence intensity $I_{v}=\sigma_{v} / U_{\text {hub }}$ (bottom)

area, which is about $0.15-0.18$ at $x / d \leq 1.5$. The maximum stream-wise turbulence intensity occurs at the upper and lower parts of the shear layers immediately behind the nacelle, where shear-produced turbulence is substantial. In the region of $x / d=0.5-1.2$, the maximum lateral and vertical turbulence intensities $\left(I_{w}\right.$ and $\left.I_{v}\right)$ are found at the hub height. Further downwind of the turbine, $I_{u}$ decays in the shear layers and it reaches a maximum at the top-tip level at about $x / d=3$. This finding is consistent with the previous measurements as well as the LES results reported by Porté-Agel et al. (2011), which found the maximum stream-wise turbulence intensity occurs at the top-tip level, about two to five rotor diameters downwind of the turbine. The significant turbulence enhancement is associated with strong wind shear and high mechanical production of turbulent kinetic energy around the top-tip level.

The variation of the turbulence intensities in the spanwise direction can be seen in Figs. 14 and 15. At $x / d=1$, the maximum values of $I_{v}$ and $I_{w}$ are about 0.18 in the middle of the rotor-swept plane. This trend agrees well with that observed in the vertical stream-wise plane (in Fig. 13b, c). However, the contours of $I_{v}$ and $I_{w}$ are not completely axisymmetric in these cross-sectional measurement planes $(x / d=1,2$ and 3$)$. Instead, the distribution of the turbulence intensities is tilted, owing to pronounced rotation in the near-wake region. With increasing downwind distance from the rotor, the magnitude of turbulence intensity decreases and the area of influence spreads out as the wake expands. At $x / d=3$, the high turbulence intensities are distributed close to the edge of the rotor-swept area. From the results presented here, it is evident that the areas of high $I_{w}$ and $I_{v}$ deviated slightly from the central vertical plane $(y=0)$ in the wake. It should be noted that we are not able to measure the spatial distribution of $I_{u}$ in the span-wise planes with the 2D2C PIV. However, Wu and Porté-Agel (2011) reported previous wind-tunnel results of $I_{u}$ measured with a cross-wire and found a similar tilting distribution, as shown here for $I_{w}$ and $I_{v}$, in the span-wise plane at $x / d=5$. Moreover, we obtained vertical profiles of $I_{u}$ and $I_{w}$ at selected downwind locations with the HWA (in Fig. 16). The maximum value of $I_{w}$ is about $67 \%$ of the maximum $I_{u}$, which occurs at $x / d=2$ and 3. This agrees with the PIV data. Also, note that the maximum turbulence intensities occur around the upper edge of the rotorswept plane. These results demonstrate the importance of all three components of turbulence intensity, $I_{u}, I_{v}$ and $I_{w}$, since they are of comparable magnitude in the near-wake region.

Furthermore, the Reynolds shear stress distribution in the vertical stream-wise plane are presented in Fig. 17. High levels of the stream-wise, wall-normal Reynolds shear stress $\left(-\overline{u^{\prime} w^{\prime}}\right)$ occur in the shear layers immediately behind the nacelle and again at the top-tip level at $x / d=2.5-3$. The distribution is very similar to that of $I_{u}$, resulting from the strong wind shear and associated mechanical production of turbulent kinetic energy. The maximum value of the Reynolds stress component $-\overline{v^{\prime} w^{\prime}}$ is about half of $-\overline{u^{\prime} w^{\prime}}$ and is also found behind the nacelle. It reduces with increasing downwind distance, except at the top-tip level (about $x / d=3$ ) where a relatively high magnitude is also found. The distribution of the Reynolds stress component $-\overline{v^{\prime} w^{\prime}}$ in the vertical span-wise planes (not shown here) is roughly symmetric with respect to the rotor axis in the near wake $(x / d=1$ and 2$)$. It should be noted that PIV results from two orthogonal views are consistent with each other. As the downwind distance from the turbine increases, the wake expands and the Reynolds shear stresses decrease. 
Fig. 14 Lateral turbulence intensity $I_{v}\left(\sigma_{v} / U_{\text {hub }}\right)$ distribution in span-wise planes at selected downwind locations. $\mathbf{a} x / d=1 ; \mathbf{b} x / d=2 ; \mathbf{c} x / d=3$ and $\mathbf{d} x / d=5$

\section{Summary and conclusions}

Wind-tunnel measurements of the turbulent wake downwind of a model wind turbine were conducted in a neutrally stratified boundary layer flow. We carried out high-resolution PIV measurements in two orthogonal views and HWA measurements at multiple downwind locations to capture the signature of coherent tip vortices and map the spatial variation of the mean flow and turbulence statistics in the near wake.

The spatial distribution of vorticity and swirling strength measured with PIV reveals the presence of top-tip vortices, which persist up to about 2-3 rotor diameters downwind of the turbine, longer than the hub/root vortices in the near wake. The power spectrum of velocity fluctuations confirms the vortex shedding with strong spectral peaks at multiples of the frequency of consecutive rotating blades at the top-tip and side-tip levels. Using neither PIV nor HWA data, can we find a similar tip vortex signature at the bottom-tip height, which is attributed to the higher background turbulence level compared to the top-tip height.
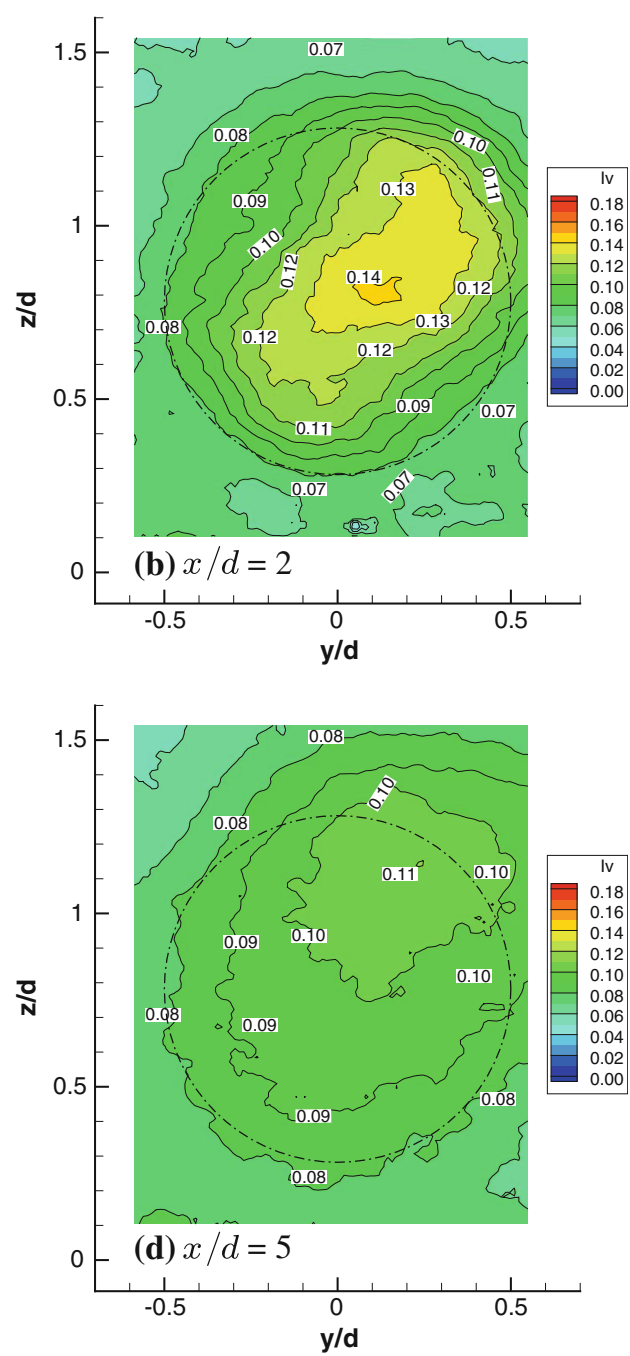

Due to the effect of the wind turbine, which extracts momentum from the flow while inducing rotation, the stream-wise velocity decreases strongly and the lateral and vertical velocities increase immediately behind the rotor. As the wake grows with increasing downwind distance from the rotor, the stream-wise velocity increases and the other velocity components decrease. From the velocity fields measured in the multiple vertical streamwise wake planes and vertical span-wise planes, it is shown that the near-wake region is characterized by high three-dimensionality, turbulence heterogeneity and strong flow rotation. Accounting for the non-uniformity and rotation of the flow induced by the turbine is important to obtain accurate simulation of wind turbine wakes, as recently shown by Wu and Porté-Agel (2011) and PortéAgel et al. (2011).

Spatial distributions of turbulence intensities and Reynolds shear stress display strong variability, with the three turbulence intensities having comparable values in the near wake. The significant turbulence enhancement at a distance of three rotor diameters is associated with strong wind 
Fig. 15 Vertical turbulence intensity $I_{w}\left(\sigma_{w} / U_{\text {hub }}\right)$ distribution in span-wise planes at selected downwind locations. $\mathbf{a} x / d=1 ; \mathbf{b} x / d=2 ; \mathbf{c} x / d=3$; and $\mathbf{d} x / d=5$
Fig. 16 Vertical profiles of the stream-wise (left) and vertical (right) turbulence intensity at selected downwind locations $(x / d=2,3,5,10$, and 20)
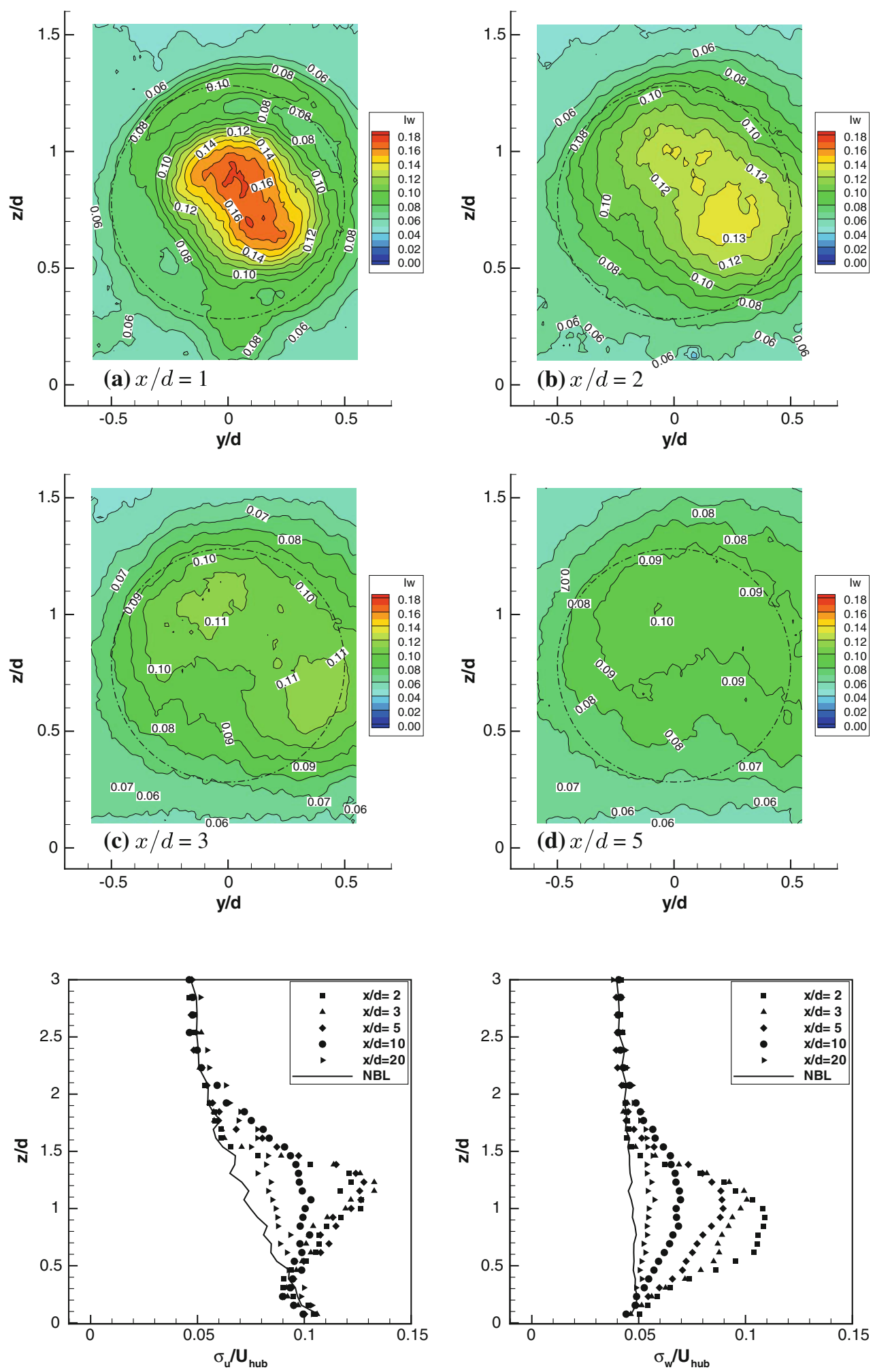

shear and high mechanical production of turbulent kinetic energy at the top-tip level.

The effect of atmospheric stability (stable and convective boundary layers) on the turbulent structure of wind turbine wakes is of great interest to the wind energy community. Currently, the effects of a convective boundary layer on the structure of wind turbine wakes are being analyzed. Additionally, future research will include the study of other factors such as the coupled effects of complex topography and boundary layer flow, as well as the 

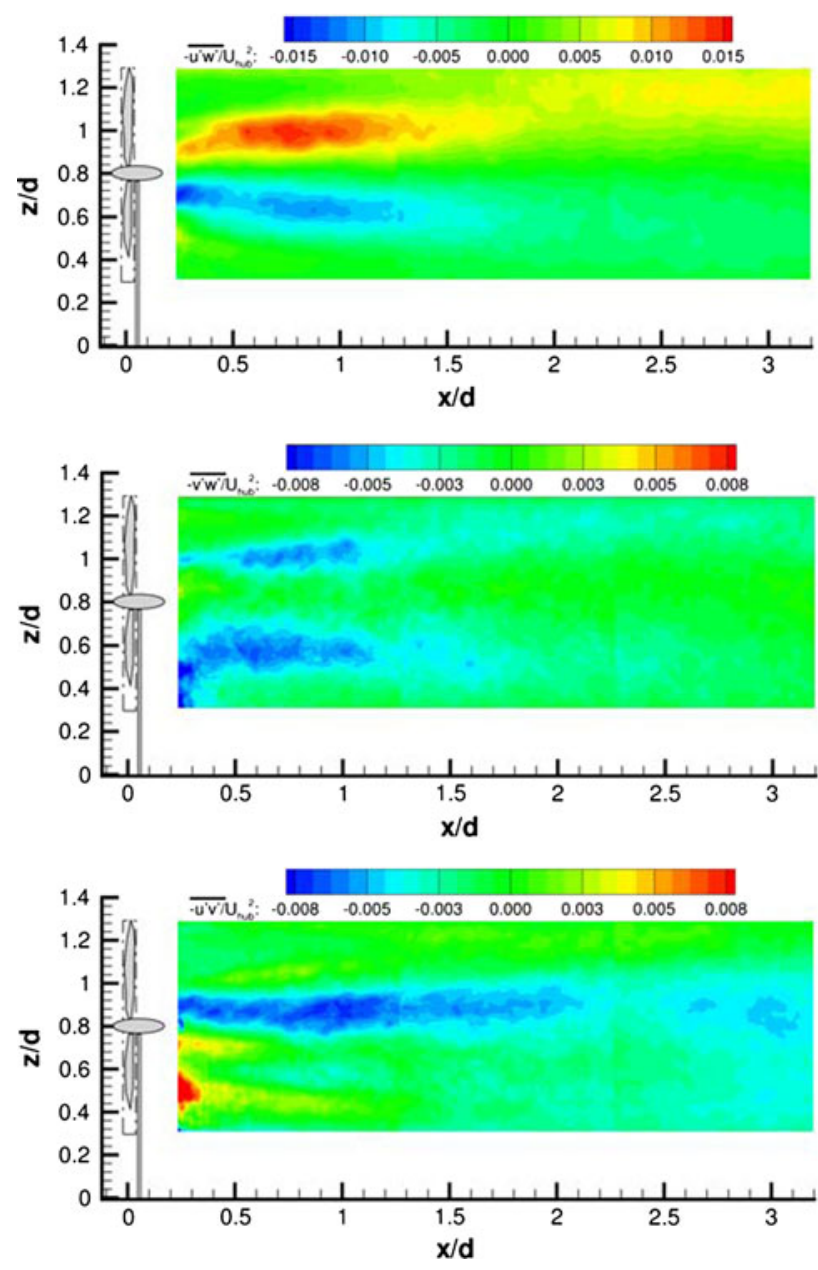

Fig. 17 Normalized Reynolds shear stresses, $-\overline{u^{\prime} w^{\prime}} / U_{\text {hub }}^{2}$ (top), $-\overline{v^{\prime} w^{\prime}} / U_{\text {hub }}^{2}($ mid $),-\overline{u^{\prime} v^{\prime}} / U_{\text {hub }}^{2}($ bottom $)$ in the central wake plane at $x / d=0-3$

interaction of multiple wakes in wind farms with various layouts/configurations.

Acknowledgments This research was supported by the Swiss National Science Foundation (grant 200021-132122), the National Science Foundation (grant ATM-0854766), NASA (grant NNG06GE256), customers of Xcel Energy through a grant (RD3-42) from the Renewable Development Fund, and the University of Minnesota Institute for Renewable Energy and the Environment. C. M. would like to acknowledge funding from NSF IGERT (Grant DGE0504195) and NASA Earth and Space Science Fellowship (Grant NNX10AN52H). Thanks also go to the research engineer James Tucker for his efforts in preparation of the experimental facility and instruments. Computing resources were provided by the University of Minnesota Supercomputing Institute and the Swiss National Supercomputing Centre.

\section{References}

Adrian RJ, Christensen KT, Liu ZC (2000) Analysis and interpretation of instantaneous turbulent velocity fields. Exp Fluids 29:275-290
Ainslie JF (1988) Calculating the flow field in the wake of wind turbines. J Wind Eng Ind Aerodyn 27:213-224

Athanassiadou M, Castro IP (2001) Neutral flow over a series of rough hills: a laboratory experiment. Boundary Layer Meteorol 101:1-30

Bingöl F, Mann J, Larsen GC (2007) Laser measurements of wake dynamics. The proceedings at EWEC, Milan

Bruun HH (1995) Hotwire anemometry, principles and signal analysis. Oxford University Press, UK

Burton T, Sharpe D, Jenkins N, Bossanyi E (2001) Wind energy handbook. 1st edn. Wiley, New York

Cal RB, Lebrón J, Castillo L, Kang HS, Meneveau C (2010) Experimental study of the horizontally averaged flow structure in a model wind-turbine array boundary layer. J Renew Sustain Energy 2:013-106

Carper MA, Porté-Agel F (2008) Subfilter-scale fluxes over a surface roughness transition. Part I: measured fluxes and energy transfer rate. Boundary Layer Meteorol 126:157-179

Chamorro LP, Porté-Agel F (2009) A wind-tunnel investigation of wind-turbine wakes: Boundary-layer turbulence effects. Boundary Layer Meteorol 132(1):129-149

Chamorro LP, Porté-Agel F (2010) Thermal stability and boundarylayer effects on wind-turbine wakes: a wind-tunnel study. Boundary-Layer Meteorol 136:515-533

Crespo A, Hernandez J, Frandsen S (1999) Survey of modelling methods for wind turbine wakes and wind farms. Wind Energy $2: 1-24$

Dobrev I, Maalouf B, Troldborg N, Massouh F (2008) Investigation of the wind turbine vortex structure. In: 14th international symposium on applications of laser techniques to fluid mechanics, Lisbon, Portugal

Gong W, Ibbetson A (2009) A wind-tunnel study of turbulent flows over model hills. Boundary-Layer Meteorol 49:113-148

Grant ALM (1992) The structure of turbulence in the near-neutral atmospheric boundary-layer. J Atmos Sci 49:226-239

Grant I, Parkin P (2000) A DPIV study of the trailing vortex elements from the blades of a horizontal axis wind turbine in yaw. Exp Fluids 28:368-376

Grant I, Parkin P, Wang X (1997) Optical vortex tracking studies of a horizontal axis wind turbine in yaw using laser-sheet, flow visualisation. Exp Fluids 23:513-519

Hancock PE, Pascheke F (2010) Wind tunnel simulations of wind turbine wake interactions in neutral and stratified wind flow. In: 10th EMS annual meeting, 10th European Conference on Applications of Meteorology (ECAM) Abstracts, September 13-17, 2010 in Zurich, Switzerland

Hansen AC, Butterfield CP (1993) Aerodynamics of horizontal-axis wind turbines. Annu Rev Fluid Mech 25:115-149

Hutchins N, Nickels TB, Marusic I, Chong MS (2009) Hot-wire spatial resolution issues in wall-bounded turbulence. J Fluid Mech 635:103-136

Jorgensen FE (1996) The computer-controlled constant temperature ameometer: aspectes of the set-up, probe calibration, data acquisition, and data collection turbulence. Meas Sci Technol 12:1378-1387

Katul GG, Chu CR, Parlange MB, Albertson JD, Ortenburger TA (1995) Low-wave-number spectral characteristics of velocity and temperature in the atmospheric surface-layer. J Geophys Res Atmos 100:14243-14255

Khurshudyan LH, Snyder WH, Nekrasov IV (1981) Flow and dispersion of pollutants over two-dimensional hills. Env Prot Agency Rpt No EPA-600/4-81-067 Research Triangle Park, NC

Larson GC, Madsen HA, Thomsen K, Larson TJ (2008) Wake meandering: a pragmatic approach. Wind Energ 11:377-395

Lebrón J, Castillo L, Cal RB, Kang HS, Meneveau C (2010) Interaction between a wind turbine array and a turbulent 
boundary layer. In: 48th AIAA aerospace sciences meeting including the new horizons forum and aerospace exposition, 4-7 January 2010, Orlando, Florida

Lu H, Porté-Agel F (2011) Large-eddy simulation of a very large wind farm in a stable atmospheric boundary layer. Phys Fluids 23, 065101:1-065101:19. doi:10.1063/1.3589857

Massouh F, Dobrev I (2007) Exploration of the vortex wake behind of wind turbine rotor. J Phys Conf Series 75, 012036:1-012036:9. doi:10.1088/1742-6596/75/1/012036

Medici D, Alfredsson PH (2006) Measurements on a wind turbine wake: $3 \mathrm{D}$ effects and bluff body vortex shedding. Wind Energy 9:219-236

Medici D, Alfredsson PH (2008) Measurements behind model wind turbines: further evidence of wake meandering. Wind Energ 11:211-217

Pedersen TF, Antoniou I (1989) Visualisation of flow through a stallregulated wind turbine rotor. Wind Eng 13:239-245

Porté-Agel F, Wu YT, Lu H, Conzemius RJ (2011) Large-eddy simulation of atmospheric boundary layer flow through wind turbines and wind farms. J Wind Eng Ind Aerodyn 99:154-168

Raffel M, Willert CE, Wereley ST, Kompenhans J (2007) Particle image velocimetry: a practical guide. 2nd edn. Springer, Berlin

Sherry M, Sheridan J, Jacono DL (2010) Horizontal axis wind turbine tip and root vortex measurements. In: 15th international symposium on applications of laser techniques to fluid mechanics, Lisbon, Portugal

Simms D, Schreck S, Hand M, Fingersh LJ (2001) NREL unsteady aerodynamics experiment in the NASA-AMES wind tunnel: a comparison of predictions to measurements. NREL/TP-50029494

Snel H, Schepers JG, Montgomerie B (2007) The MEXICO project (model experiments in controlled conditions): the database and first results of data processing and interpretation. J Phys Conf Ser 75:012-014

Sørensen JN (2011) Aerodynamic aspects of wind energy conversion. Annu Rev Fluid Mech 43:427-448

Vermeer LJ (2001) A review of wind turbine wake research at tudelft. In: Proceedings of ASME wind energy symposium, ASME, New York, AIAA-2001-0030, vol 39, pp 103-113

Vermeer LJ, Sørensen JN, Crespo A (2003) Wind turbine wake aerodynamics. Progr Aero Sci 39:467-510

Whale J, Papadopoulos KH, Anderson CG, Helmis CG, Skyner DJ (1997) A study of the near wake structure of a wind turbine comparing measurements from laboratory and full-scale experiments. Sol Energy 56:621-633

Whale J, Anderson CG, Bareiss R, Wagner S (2000) An experimental and numerical study of the vortex structure in the wake of a wind turbine. J Wind Eng Ind Aerodyn 84:1-21

Wu YT, Porté-Agel F (2011) Large-eddy simulation of wind-turbine wakes: evaluation of turbine parametrizations. Boundary-Layer Meteorol 132:129-149

Zhou J, Adrian RJ, Balachandar S, Kendall TM (1999) Mechanisms for generating coherent packets of hairpin vortices in channel flow. J Fluid Mech 387:353-359 